\section{Adaptação transcultural para o Brasil da Exercise Benefits/Barriers Scale (EBBS) para aplicação em idosos: uma avaliação semântica}

\author{
Cross-cultural adaptation of the Exercise Benefits/ \\ Barriers Scale (EBBS) for application in elderly \\ Brazilians: preliminary version
}

\author{
${ }^{1}$ Centro de Ciências da \\ Saúde, Universidade de \\ Fortaleza, Fortaleza, Brasil. \\ 2 Faculdade de Farmácia, \\ Odontologia e Enfermagem, \\ Universidade Federal do \\ Ceará, Fortaleza. \\ ${ }_{3}$ Centro de Ciências da \\ Saúde, Universidade \\ Estadual do Ceará, Fortaleza, \\ Brasil. \\ Correspondência \\ J. F. Victor \\ Departamento de \\ Enfermagem, Centro \\ de Ciências da Saúde, \\ Universidade de Fortaleza. \\ Av. Rui Barbosa 2700, \\ apto. 1401, Fortaleza, CE \\ 60115-222, Brasil. \\ janainavictor@uol.com.br
}

\begin{abstract}
This article describes the cross-cultural adaptation of the Exercise Benefits/Barriers Scale (EBBS) to the Portuguese language and its application in elderly Brazilians. The EBBS was originally developed in the United States to identify perceptions concerning the barriers to (and benefits of) physical exercise, and thus to determine factors affecting this practice. Cross-cultural adaptation involved the following phases: initial translation, summary of the translation, retranslation into the original language (back-translation), review by an expert panel to evaluate semantic, idiomatic, experimental, and conceptual equivalence, and pre-test of the final version with 30 elderly subjects. The EBBS cross-cultural adaptation allowed proposing a preliminary Portuguese-language version. However, to ensure that the cross-cultural equivalence between the original version and the translation has been fully achieved, it is necessary to compare the psychometric properties of the Portuguese version and the original instrument, a crucial task that is now being performed and will soon be published.
\end{abstract}

Motor Activity; Aged; Validation Studies
Janaina Fonseca Victor 1

Lorena Barbosa Ximenes 2

Paulo Cesar de Almeida 3

Durante o envelhecimento, o organismo é objeto de alterações fisiológicas em sua capacidade física, principalmente, alterações cardiovasculares e musculoesqueléticas 1. Em idosos, as evidências clínicas e epidemiológicas revelam que a prática de atividade física regular tem efeito positivo na promoção da saúde, possibilitando prevenção e minimização dos efeitos deletérios do envelhecimento $1,2,3$.

A prática de atividade física em idosos melhora a densidade mineral óssea, a força, a flexibilidade articular, a glicemia, o perfil lipídico, a auto-estima e a autoconfiança, além de auxiliar no controle do peso corporal, da pressão arterial, da função pulmonar, do equilíbrio, da marcha, permitindo menor dependência para realização das atividades diárias e diminuindo a incidência de quedas, o risco de fraturas, a ansiedade e a depressão. Todos esses benefícios são incontestáveis quanto à repercussão na qualidade de vida dos que a praticam 2,4,5.

Em idosos, em particular, iniciativas mundiais para a promoção da saúde, mediante a prática de atividade física, são desenvolvidas pela Organização Mundial da Saúde (OMS) desde 1996, quando elaborou o protocolo The Heiderberg Guidelines for Promoting Physical Activity Among Older Persons 6, o qual traz evidências científicas dos benefícios da prática de atividade física. Em 1999, a OMS instituiu o Ano Internacional do Idoso, e criou a Internacional Society for Aging and Physical Activity (ISAPA). 
O American College of Sports Medicine Position Stand publicou em 1998, o documento Exercise and Physical Activity for Older Adults 7, trazendo as mudanças observadas na função cardiovascular, na força muscular, flexibilidade, estabilidade postural e capacidade funcional dos idosos com a atividade física.

No Brasil, em 1999, a Sociedade Brasileira de Medicina do Esporte, em parceria com a Sociedade Brasileira de Geriatria e Gerontologia, elaboraram o texto sobre atividade física e saúde dos idosos, cujo propósito é ampliar a recomendação da prática de atividade física pelos profissionais de saúde que lidam com indivíduos idosos 8. Em 2006, o Ministério da Saúde brasileiro propôs a Política Nacional de Promoção da Saúde (PNPS), que prioriza a prática corporal e a atividade física, tanto para a população como um todo quanto para grupos vulneráveis, como os idosos 9 .

É mister esclarecer que as expressões atividade física e exercício físico não se apresentam como sinônimos, pois a primeira se refere a qualquer movimento do corpo produzido por músculos esqueléticos e que resulta em dispêndio de energia, enquanto o exercício é a atividade planejada, realizada de forma repetitiva e feita com algum propósito 2 .

Embora ciente da diferença das expressões, neste estudo concorda-se com Pitanga \& Lessa ${ }^{10}$, que definem atividade física como movimento corporal produzido pela musculatura esquelética que resulte em gasto energético, tendo componentes e determinantes de ordem biológica, psicossocial, cultural e comportamental, podendo ser exemplificada por jogos, lutas, danças, esportes e exercícios físicos.

É imprescindível ressaltar ainda que a inatividade em pessoas idosas acontece, muitas vezes, por determinações sócio-culturais, e não verdadeiramente por uma incapacidade funcional. Os comportamentos conferidos aos idosos referem-se à dependência e imobilidade, com baixo nível de atividade física. Sabe-se, entretanto, que muitas das alterações funcionais observadas nos idosos são conseqüências da inexistência de estímulos e não essencialmente das alterações atribuídas ao envelhecimento 11,12.

Dentre os múltiplos fatores que levam à inatividade, o desconhecimento sobre como se exercitar, as finalidades de cada exercício, limitações de grupos específicos e percepções distorcidas em relação à atividade física são apontados como possíveis fatores para o sedentarismo 13 .

O conhecimento sobre os benefícios da atividade física não implica necessariamente adoção de um estilo de vida fisicamente ativo, todavia, sem o conhecimento e percepção adequados sobre este tema é improvável que atitudes de mudança no padrão de comportamento sejam postas em prática. Nesse sentido, estudos realizados acerca da relação entre o conhecimento sobre atividade física e sua prática, evidenciaram o fato de que, quando se conhecem as percepções a respeito de atividade física em populações mais vulneráreis, como os idosos, as intervenções, principalmente, no âmbito primário, são mais efetivas 4,5,14.

Uma opção para os profissionais de saúde no entendimento das facilidades e dificuldades para a prática de atividade física é a utilização de escala de medida que possa avaliar o conhecimento que as pessoas têm sobre este tema. $\mathrm{O}$ uso de escalas fornece uma medida objetiva e psicometricamente rigorosa. No entanto, após vasto levantamento bibliográfico, na literatura nacional, realizado nos principais bancos de dados, nenhum instrumento foi encontrado. Há instrumentos que medem o nível de atividade física das pessoas, mas não o conhecimento sobre o assunto.

Entretanto, encontra-se na literatura internacional o instrumento de avaliação psicométrica denominado Exercise Benefits/Barriers Scale (EBBS), desenvolvido por Sechirist et al. 15. Ao aplicar o instrumento pode-se identificar tanto a percepção sobre as barreiras encontradas para ser fisicamente ativo quanto os benefícios relacionados à prática de exercícios. A EBBS foi elaborada com base no modelo teórico denominado Modelo de Promoção da Saúde de Nola J. Pender. Relevante destacar ainda, que esse instrumento não foi desenvolvido especificamente para idosos, mas para aplicação em adultos.

Neste estudo, estabeleceu-se como objetivo a adaptação transcultural para o Brasil da EBBS para aplicação em idosos. A decisão pela tradução do instrumento para idosos deu-se pela importância dessa atividade para esse grupo etário, além de não existir na literatura instrumento específico.

\section{Exercise Benefits/Barriers Scale}

A EBBS é um instrumento com 43 itens, sendo 14 da barrier scale e 29 da benefits scale. Ele pode ser aplicado conjunta ou separadamente. A escala apresenta opções de resposta do tipo Likert com os escores dos itens variando de 1 a 4: strongly agree (4), agree (3), desagree (2), strongly desagree (1). Quando usadas juntas, a EBBS apresenta escore reverso aos itens da barriers scale podendo variar de escores de 43 a 172. Quando usadas isoladamente, entretanto, a maior pontuação é para a benefits scale, que varia de escores 29 a 116, e para barriers scale varia de escores 14 a 56, repre- 
sentando melhor percepção sobre os benefícios e as barreiras, respectivamente 15 .

A confiabilidade da EBBS foi testada pela análise da consistência interna (alfa de Cronbach), que foi de 0,954 para benefitis scale e 0,866 para barriers scale. A confiabilidade teste-reteste foi de 0,89 no instrumento total, sendo 0,89 para benefits scale e 0,77 para barriers scale 15 .

\section{Métodos}

Os procedimentos metodológicos para tradução e adaptação da EBBS seguiram os estágios do processo proposto por Beaton et al. 16, envolvendo a adaptação de itens, instruções e opções de repostas da escala. A proposta compreendeu cinco fases: tradução inicial, síntese da tradução, tradução de volta à língua de origem (back-translation), revisão por um comitê de juízes e pré-teste da versão final. Esse processo de adaptação é o refinamento da versão anteriormente proposta por Guillemin et al. 17 .

A autorização para o uso do instrumento foi obtida por meio de correio eletrônico junto à Professora Doutora Nola J. Pender.

\section{Fase I: tradução inicial}

Foram realizadas duas traduções independentes para a língua portuguesa. O tradutor um ou (T1) não era da área de saúde (corretora de imóveis), tem dupla nacionalidade (americana e brasileira), é residente nos Estados Unidos, é bilíngüe e filha de brasileiros; foi informada do objetivo da tradução. O segundo tradutor ou (T2) é profissional de saúde (médica) com fluência em inglês e não foi informada do objetivo da tradução.

\section{Fase II: síntese das traduções}

Procedeu-se a sintetização dos resultados das traduções, trabalhando com o instrumento original, bem como, com as traduções do T1 e T2. Essa síntese foi cuidadosamente registrada quanto a cada item do instrumento. Após os ajustes entre as duas traduções, o instrumento foi chamado de T12, sendo utilizado na próxima fase.

Fase III: tradução de volta à língua de origem (back-translation)

O procedimento adotado nesta etapa consistiu no envio do instrumento traduzido (T12) para a sua língua original, etapa denominada de back-translation, por dois tradutores, os quais não tiveram conhecimento dos objetivos da back-translation e não foram informados quanto ao que estava sendo feito. O back-translation foi realizado por dois professores de inglês, que já estudaram e moraram nos Estados Unidos, e foram chamados de B1 e B2, em referência ao termo back-translation. Após a realização do B1 e B2, os instrumentos foram encaminhados à próxima fase.

\section{Fase IV: revisão por um comitê}

Todos os instrumentos produzidos até esta fase - as duas traduções T1 e T2, a tradução T12 e as duas versões do back-translation (B1 e B2), além da versão original - foram examinados por um comitê de juízes formado por um educador físico, mestre em epidemiologia que desenvolve atividades com idosos; uma enfermeira, mestre em saúde pública e coordenadora de um grupo de idosos; e dois professores de língua inglesa.

Enviou-se a cada membro do comitê os instrumentos há pouco citados, como também os aspectos a serem avaliados, a saber: (a) equivalência semântica - avaliação gramatical e do vocabulário; (b) equivalência idiomática - a formulação de expressões equivalentes para o idioma português, quando a tradução de termos e expressões idiomáticas for de tradução difícil; (c) equivalência experimental - a tradução deve utilizar termos coerentes com a experiência vivida pela população à qual se destina; (d) equivalência conceitual - os conceitos traduzidos devem ser explorados e os eventos experimentados pela população brasileira.

Após a avaliação pelos membros do comitê, procedeu-se à revisão de todas as anotações realizadas para a síntese do instrumento final; realizou-se uma reunião com os membros do comitê para avaliação da versão final. O instrumento foi, então, aprovado para ser utilizado no pré-teste.

\section{Fase V: pré-teste}

Nesta fase, buscou-se a equivalência das versões original e final, a fim de que fosse verificada sua compreensão pela população-alvo.

O pré-teste foi realizado com trinta idosos e após o preenchimento da escala foi-lhes solicitado que avaliassem os itens do instrumento traduzido quanto à sua compreensão, pertinência, aprovação e relevância cultural. Dadas as características dos sujeitos quanto à escolaridade, aplicou-se a escala a vinte idosos e dez responderam à escala sozinhos. Após a aplicação, cada sujeito relatou suas impressões sobre o instrumento e suas respostas para cada item. 


\section{Resultados}

Os itens da escala original e da versão traduzida e adaptada podem ser vistos na Tabela 1. A escala de graduação de respostas da EBBS foi considerada não equivalente; as opções de respostas na escala original são: strongly agree, agree, disagree, strongly disagree (a palavra strongly que significa fortemente, foi alterada para "totalmente"). Assim, as opções de respostas da escala passaram a ser: concordo totalmente, concordo, discordo e discordo totalmente.
As adaptações mais relevantes foram as substituições dos termos exercise que para o português seria exercício físico, e exercing (praticar exercício) por atividade física, em todos os itens da escala. Os juízes sugeriram a não-utilização do termo exercício e sim a expressão atividade física, uma vez que estes não se apresentam conceitualmente equivalentes no idioma.

No Brasil, as pessoas geralmente associam o termo exercício ao uso de equipamentos, como, por exemplo, a musculação, além de ginástica e treinamentos com instrutores. Atividades como

Tabela 1

Versão original e versão traduzida e adaptada dos itens para o Brasil da Exercise Benefits/Barriers Scale (EBBS).

Versão original

1. I enjoy exercise

2. Exercise decreases feelings of stress and tension for me

3. Exercise improves my mental health

4. Exercise takes too much of my time

5. I will prevent heart attacks by exercising

6. Exercise tires me

7. Exercise increases my muscle strength

8. Exercise give me a sense of personal accomplishment

9. Places for me to exercise are too far away

10. Exercising makes me feel relaxes

11. Exercising lets me have contact with friends and persons I enjoy

12. I am too embarrassed to exercise

13. Exercising will keep me from having high blood pressure

14. It costs too much money to exercise

15. Exercising increases my level of physical fitness

16. Exercise facilities to not have convenient schedule for me

17. My muscle tone is improve with exercise

18. Exercising improves functioning of my cardiovascular system

19. I am fatigued by exercise

20. I have improved feeling of well being from exercise

21. My spouse (or significant other) does not encourage exercising

22. Exercise increases my stamina

23. Exercise improves my flexibility

24. Exercise takes too much time from family relationships

25. My disposition is improved by exercise

26. Exercising helps me sleep better at night

27. I will live longer if I exercise
Versão traduzida e adaptada

Gosto de atividade física

Praticar atividade física diminui sentimentos de stress e preocupação

Atividade física melhora a saúde mental (sentir-se alegre e bem

emocionalmente)

Praticar atividade física toma muito tempo

Irei prevenir ataque do coração se praticar atividade física

Atividade física causa cansaço

Atividade física aumenta a força dos músculos (capacidade de empregar

um esforço - ex.: empurrar um móvel, levantar um objeto)

Atividade física proporciona sensação de realização pessoal

(estar bem comigo mesmo)

Os locais para praticar atividade física são distantes

Atividade física causa relaxamento

Atividade física facilita ter mais contatos com amigos e pessoas de

quem gosto

Sinto vergonha para praticar atividade física

A atividade física protege de ter pressão alta

Praticar atividade física custa caro

Atividade física melhora a aptidão física (habilidades motoras, capacidade

e habilidade de caminhar, subir escada, varrer a casa)

Não tenho horário conveniente para praticar atividade física

Atividade física melhora o tônus muscular (músculos preparados para

um movimento de contração)

Atividade física melhora o funcionamento do coração e vasos sanguíneos

Ao praticar atividade física sinto cansaço

Posso melhorar sentimentos de bem-estar (ficar mais feliz) com a

atividade física

Meu/minha esposo(a) ou as pessoas importantes para mim não me

incentivam a fazer atividade física

Atividade física melhora a flexibilidade (capacidade de realizar movimentos com as articulações, levantar o braço, curvar o corpo)

Praticar atividade física toma muito tempo de estar com a família

Atividade física melhora a disposição (ânimo para fazer as coisas)

Praticar atividade física ajuda a dormir melhor à noite

Irei viver mais se praticar atividade física

(continua) 
Tabela 1 (continuação)

\begin{tabular}{|c|c|}
\hline Versão original & Versão traduzida e adaptada \\
\hline 28. I think people in exercise clothes look funny & Acho que as roupas para praticar atividade mostram muito o corpo \\
\hline 29. Exercise helps me decrease fatigue & Atividade física reduz o cansaço muscular \\
\hline 30. Exercising is a good way for me to meet new people & Atividade física é uma boa maneira para conhecer novas pessoas \\
\hline 31. My physical endurance is improved by exercise & $\begin{array}{l}\text { Atividade física melhora a resistência física (conseguir caminhar por 30min } \\
\text { ou mais) }\end{array}$ \\
\hline 33. My family members do not encourage me to exercise & As pessoas da família não me incentivam a praticar atividade física \\
\hline 34. Exercise increases my mental alertness & Atividade física melhora a agilidade mental (rapidez do pensamento) \\
\hline 35. Exercise allows me to carry out normal activities without & Praticar atividade física permite a realização das atividades do \\
\hline becoming tired & dia-a-dia sem sentir cansaço \\
\hline 36. Exercise improves the quality of my work & Atividade física melhora a qualidade das atividades que faço \\
\hline 37. Exercise take too much time from my family responsibilities & Atividade física toma muito tempo das responsabilidades familiares \\
\hline 39. Exercise increases my acceptance by others & Praticar atividade física aumenta a minha aceitação pelas pessoas \\
\hline 40. Exercise s hard work for me & Praticar atividade física é uma tarefa difícil \\
\hline 41. Exercise improves overall body functioning for me & Atividade física melhora todo o funcionamento do corpo \\
\hline 42. There are too few places for me to exercise & Existem poucos lugares para praticar atividade física \\
\hline 43. Exercise improves the way my body looks & Praticar atividade física melhora a aparência do corpo \\
\hline
\end{tabular}

caminhadas não são consideradas por muitas pessoas como um tipo de exercício. Esse fato foi confirmado durante a realização do pré-teste. Ao iniciar a aplicação do instrumento, perguntavase: "Você faz exercícios?". Todos os sujeitos respondiam que "não", mesmo os que realizavam caminhadas. Quando a pergunta era substituída por: "Você faz atividade física?", a caminhada era citada. No intuito de garantir a equivalência cultural, os termos foram alterados.

Os itens 03, 07, 08, 15, 17, 20, 22, 23, 31, 32 e 34 , além de traduzidos, foram exemplificados. A utilização de exemplos para esses itens foi recomendação dos sujeitos durante o pré-teste. $\mathrm{O}$ comitê de juízes julgou imprescindível essa sugestão para facilitar o entendimento da escala pela população do estudo.

Os itens 07, 15, 17, 22, 23 e 31 são termos específicos da Educação Física e foram exemplificados por dois educadores físicos (não-membros do comitê). O item 07 ("exercise increase my muscle strength") foi traduzido para "atividade física aumenta a força dos músculos”. Exemplo: capacidade de empregar um esforço, como empurrar um objeto.

Em relação à expressão muscle tônus (tônus muscular) do item 17, dois exemplos foram sugeridos (músculos mais endurecidos ou músculos preparados para realizar um movimento de contração). Optou-se pelo segundo exemplo após consulta à literatura sobre o tema.

Os itens 15, 22 e 31 trazem palavras como physical fitness, stamina, physical endurance.
Tais expressões estão relacionadas à capacidade funcional das pessoas em relação a exercícios, não apresentando clara correlação com o idioma português, sendo de compreensão difícil para a população do estudo. Os itens foram traduzidos pelo comitê e exemplificados pelos educadores físicos, respectivamente, como aptidão física (capacidade para caminhar, subir escada); condicionamento físico (capacidade de subir escada, dançar, varrer a casa sem se cansar) e resistência física (conseguir caminhar por trinta minutos ou mais). Relevante é destacar que os itens foram exemplificados levando-se em consideração as características da população do estudo (idosos), e não retratam todas as possibilidades para exemplificação dos vocábulos.

Segundo os educadores físicos, os itens 15 e 22, quando traduzidos para o idioma português, apresentaram o mesmo significado (resistência) e foram considerados itens ambíguos. Fato esse comprovado em consulta a três dicionários do tipo inglês-português, em que todas as traduções encontradas para as palavras stamina e endurance são absolutamente iguais 18,19,20.

Em virtude desse impasse, buscou-se na literatura estudos envolvendo adaptação transcultural de instrumentos de medidas para observar a ocorrência de situação análoga, todavia, nos estudos avaliados, nenhum pesquisador relatou a ocorrência de itens ambíguos 21,22,23,24,25,26,27, 28,29,30,31,32,33,34,35,36,37.

Essa situação ímpar vivenciada pelo comitê colocou em questão as seguintes opções: manter 
dois itens iguais ou sugerir a retirada de um deles. Entretanto, nos estudos que envolvem adaptação transcultural não é comum a retirada de itens sem a avaliação das propriedades psicométricas, uma vez que isto poderá comprometê-la ${ }^{38}$. Devido à particularidade dos termos em questão, nenhuma outra palavra ou expressão poderia ser utilizada sem envolver mudança no sentido e o no conteúdo dos itens da escala original. Assim, optou-se pela retirada do item 22 , pois resistência física é uma expressão mais conhecida do que condicionamento físico.

No item 23, a palavra flexibility, em português flexibilidade, foi exemplificada como capacidade de realizar movimentos com as articulações, curvar o corpo, levantar o braço.

Nos itens 04, 09, 12, 14, 16, 19, 22, 23, 27, 34, 38,39 e 41 acrescentou-se a palavra "praticar" à expressão atividade física no sentido de dar uma conotação de ação aos itens.

Durante a fase de pré-teste da escala traduzida, os idosos sugeriram a retirada dos pronomes da maioria dos itens. Segundo os idosos, o item passava a ter uma instrução afirmativa mais compreensiva especialmente quando a escala era aplicada verbalmente.

Essa observação na tradução corrobora as recomendações quanto à versão de instrumentos de medida que devem incluir sentenças curtas, palavras-chave, voz ativa e termos específicos 28 .

O item 18 traz a expressão cardiovascular system, que traduzida é sistema cardiovascular, que foi alterada após o pré-teste para coração e vasos sanguíneos, pois os idosos solicitavam exemplos do que seria sistema cardiovascular.

O item 28 "I think people in exercise clothes look funny" foi alterado, pois sua tradução é "eu acho que pessoas em roupas de exercícios parecem engraçadas", trazendo uma situação que não tem significado no contexto brasileiro. As roupas utilizadas para praticar atividades físicas pela população dos Estados Unidos são diferentes das utilizadas no Brasil, especialmente por questões climáticas. Nesse item foi necessário realizar a equivalência experimental, o que necessitou explorar aspectos relacionados às roupas que representassem a percepção de brasileiros na prática de atividades físicas. As roupas utilizadas no Brasil precisam ser leves e em sua maioria justas ao corpo; relevante destacar que esta realidade não se aplica a todas as regiões brasileiras, no entanto, este estudo foi desenvolvido no Nordeste, onde as temperaturas são altas durante todo o ano.

Durante o pré-teste, foi solicitado que os idosos opinassem sobre as roupas utilizadas para praticar atividades físicas, sendo observado se estas representam estímulo ou obstáculo. A re- ação dos idosos em relação a esse item foi de receio em evidenciar a silhueta; assim como no item da versão original, nesta população percebeu-se que a vestimenta influenciou de forma negativa na prática de atividades físicas. Para o item 28 os idosos sugeriram "As roupas para praticar atividade física mostram muito o corpo". A sugestão foi aprovada pelo comitê, pois embora o vocabulário tenha sido alterado buscou-se preservar a equivalência experimental e cultural.

O item 35 traz a expressão normal activities, cuja tradução coincide com atividades normais. Durante o pré-teste, muitos idosos não entendiam o item e sugeriram que fosse exemplificado o que são "atividades normais". O item foi então alterado para atividades do dia-a-dia. O comitê julgou não haver prejuízo com a mudança.

$\mathrm{O}$ item 36 "exercise improves the quality of my work" apesar de corresponder, em português, a exercício melhora a qualidade do meu trabalho, foi traduzido para "atividade física melhora a qualidade das atividades que faço”, pois a palavra trabalho, para o português, tem uma conotação de emprego, cargo, função, e haja vista que muitos idosos são aposentados, tendo-se, então, optado por atividade, o que engloba outras tarefas não necessariamente relacionadas a trabalho.

Após a tradução e adaptação transcultural, a escala ficou com 42 itens que foram então renumerados. Com a saída do item 22, o valor total dos escores da versão traduzida da EBBS foi alterado, porquanto na escala original era de 43 a 172, na versão traduzida e adaptada ficou de 42 a 168 .

\section{Discussão}

Muitos autores realizaram adaptação transcultural de instrumentos, contudo, ainda não há consenso quanto à melhor metodologia a ser adotada 38,39,40,41. O modelo de adaptação transcultural seguido neste estudo foi selecionado por ser abrangente e por exigir a explicitação pormenorizada dos passos realizados.

A adaptação transcultural da EBBS foi objeto de mudanças relacionadas, principalmente, à redação das instruções dos itens que traziam termos de tradução difícil, o que não permitiu uma versão considerada simétrica. Essas mudanças envolveram exemplificações e substituições de termos e expressões, cujo objetivo principal foi facilitar a compreensão dos itens do instrumento pelos sujeitos do estudo e assim assegurar a equivalência transcultural.

Vale registrar que a adaptação transcultural não deve considerar, apenas, as diferenças entre os países ou seus idiomas, deve-se considerar 
também a realidade local ou regional, analisando-se o quanto se ganha com a aproximação cultural e o quanto se perde com a generalização e comparabilidade $38,41,42$.

Neste estudo a realização da adaptação transcultural da EBBS demandou muito mais do que a combinação de palavras e sentenças, pois foram necessárias importantes adequações em sua estrutura original. Alguns dilemas merecem ser pontuados como: as dificuldades relacionadas à complexidade e especificidade de palavras e expressões próprias da Educação Física, e o fato do instrumento não ter sido desenvolvido especificamente para idosos, estes eventos potencializaram as dificuldades no processo de tradução e trouxeram elementos para discussão de estudos futuros. É importante destacar, ainda, que a maioria $(66,6 \%)$ dos idosos não sabia ler e escrever. Pode-se considerar fator atenuante para esses eventos a existência de um educador físico no comitê de revisão e a participação efetiva dos idosos na etapa do pré-teste, na qual cada item foi minuciosamente explorado para ser compreendido.

É relevante esclarecer que os dilemas surgidos enriquecem as experiências de adaptação de instrumentos e possibilitam novos debates 43 , pois, muitas vezes, são as limitações que contribuem para o incremento e descobertas de novas pesquisas. Sobre as facilidades na adaptação transcultural, alguns autores afirmam que a tradução torna-se simples quando os itens do instrumento são de tradução fácil e inteiramente aplicáveis à realidade brasileira $32,43,44$.

A partir da adaptação transcultural da EBBS foi possível propor uma primeira versão em português. No entanto, para se aceitar com garantia que a equivalência transcultural tenha sido inteiramente conseguida, faz-se necessária a comparação entre as propriedades psicométricas da versão em português e do instrumento original, uma tarefa primordial já em realização.

Para avaliação das propriedades psicométricas da versão traduzida da EBBS serão utilizados para analise da confiabilidade o alfa de Cronbach e o teste-reteste, já a validade será analisada pela "validade de construto" por meio da investigação de associações entre os resultados da EBBS e as características sócio-demográficas (idade, escolaridade, estado civil, ocupação, com quem reside e renda), clínicas (índice de massa corporal - IMC, nível glicêmico, patologias crônicas degenerativas) e de estilo de vida (indivíduo ativo, sedentário, prática de atividades físicas regulares). O intuito de tal procedimento será avaliar se essas variáveis interferem no resultado da EBBS.

Por fim, é importante pontuar que a versão traduzida deve ser apreciada por profissionais interessados, já que o aperfeiçoamento do instrumento adaptado depende de discussões e críticas contínuas. 


\section{Resumo}

Este artigo descreve a adaptação transcultural da Exercise Benefits/Barriers Scale (EBBS) para o idioma português para aplicação em idosos. A EBBS foi originalmente desenvolvida nos Estados Unidos com o objetivo de identificar a percepção quanto às barreiras e benefícios da prática de exercícios e, assim, conhecer os fatores que interferem nesta prática. O processo de adaptação transcultural envolveu as seguintes fases: tradução inicial; síntese da tradução; tradução de volta à língua de origem (back-translation); revisão por um comitê de juízes para avaliar a equivalência semântica, idiomática, experimental e conceitual; $e$ pré-teste da versão final com trinta idosos. A partir da adaptação transcultural da EBBS foi possível propor uma versão preliminar em português. No entanto, para se aceitar com garantia que a equivalência transcultural entre o original e a versão traduzida tenha sido inteiramente conseguida, faz-se necessária a comparação entre as propriedades psicométricas da versão em português e do instrumento original, uma tarefa primordial que já está sendo realizada e oportunamente será apresentada.

Atividade Motora; Idoso; Estudo de Validação

\section{Referências}

1. Matsudo SM, Matsudo VKR, Barros Neto TL. Impacto do envelhecimento nas variáveis antropométricas, neuromotoras e metabólicas da aptidão física. Rev Bras Ciênc Mov 2000; 8:21-32.

2. Mcardle W, Katch F, Kacth V. Fundamentos de fisiologia do exercício. 2a. Ed. Rio de Janeiro: Editora Guanabara Koogan; 2002.

3. Stein R, Ribeiro JP. Atividade física e saúde. In: Duncan B, Schmidt M, organizadores. Bases clínico-epidemiológicas das condutas clínicas. Medicina ambulatorial: condutas clínicas em atenção primária. 2ạ. Ed. Porto Alegre: Editora Artes Médicas Sul; 2004. p. 508-15.

4. Schoenfelder DP, Rubestein LM. An exercise program to improve fall-related outcomes in elderly nursing home residents. Appl Nurs Res 2004; 17:21-31.

5. Resnick B. Health promotion practices of the older adult. Public Health Nurs 2000; 17:160-8.

6. Word Health Organization. The Heidelberg guidelines for promoting physical activity among older persons. Geneva: Word Health Organization; 1996.

7. American College of Sports Medicine Position Stand. Exercise and physical activity for older adults. Med Sci Sports Exerc 1998; 30:992-1008.

8. Nóbrega AC, Freitas EV, Oliveira MAB, Leitão MB, Lazzoli JK, Nahas RM, et al. Posicionamento oficial da Sociedade Brasileira de Medicina do Esporte e da Sociedade Brasileira de Geriatria e Gerontologia: atividade física e saúde no idoso. Rev Bras Med Esporte 1999; 5:207-11.

\section{Colaboradores}

J. F. Victor, L. B. Ximenes e P. C. Almeida foram responsáveis pelo processo de coleta e análise dos dados, pelo acompanhamento da etapa de adaptação transcultural da Exercise Benefits Barriers Scale (EBBS), pela discussão teórica sobre o tema e pela elaboração do texto do artigo.

9. Ministério da Saúde. Política Nacional de Promoção da Saúde. Brasília: Ministério da Saúde; 2006.

10. Pitanga FJG, Lessa I. Prevalência e fatores associados ao sedentarismo no lazer em adultos. Cad Saúde Pública 2005; 21:870-7.

11. Geis PP. Atividade física e saúde na terceira idade: teoria e prática. 5ạ. Ed. Porto Alegre: Editora Artmed; 2003

12. Schutzer KA, Graves BS. Barriers and motivations to exercise in older adults. Prev Med 2004; 39:1056-61.

13. Domingues MR, Araújo CLP, Gigante DP. Conhecimento e percepção sobre exercício físico em uma população adulta urbana do sul do Brasil. Cad Saúde Pública 2004; 20:204-15.

14. Conn VS, Tripp-Reimer T, Maas ML. Older women and exercise: theory of planned behavior beliefs. Public Health Nurs 2003; 20:153-63.

15. Sechirist K, Walker SN, Pender NJ. Development and psychometric evaluation of the Exercise Benefits/Barriers Scale. Res Nurs Health 1987; 10: 357-65.

16. Beaton D, Bombardier C, Guillemin F, Ferraz MB. Recommendations for the cross-cultural adaptation of health status measures. Rosemont: American Academy of Orthopaedic Surgeons; 1998.

17. Guillemim F, Bombadier C, Beaton D. Cross-cultural adaptation of health-related quality of life measures: literature review and proposed guidelines. J Clin Epidemiol 1993; 46:1417-32. 
18. Konder WR. English dictionary for Portuguese speakers. Rio de Janeiro: Editora Longman; 1982.

19. Aliandro H. Dicionário inglês-português. Rio de Janeiro: Editora Ao Livro Técnico; 1984.

20. Martim B, Buede-Flecher A, Ferreira MP. Dicionário Oxford escolar para estudantes brasileiros de inglês. 68a Ed. Oxford: Oxford University Press; 1996.

21. Guanilo MEE. "Burns Specific Pain Anxiety Scale - BSPAS”: adaptação transcultural e validação preliminar. [Dissertação de Mestrado]. Ribeirão Preto: Escola de Enfermagem de Ribeirão Preto, Universidade de São Paulo; 2005.

22. Galindo EMC. Tradução, adaptação e validação do Eating Behaviours and Body Image Test (EBBIT) em crianças do sexo feminino na cidade de Ribeirão Preto-SP [Dissertação de Mestrado]. Ribeirão Preto: Escola de Enfermagem de Ribeirão Preto, Universidade de São Paulo; 2005.

23. Ferreira AFB. Tradução para a língua portuguesa e validação do questionário da saúde dos pés Foot Health Status Questionnaire (FHSQ) [Tese de Doutorado]. São Paulo: Faculdade de Medicina, Universidade de São Paulo; 2005.

24. Tamanini JTN, Dambros M, D'Acona CAL, Palma PCR, Netto Jr. NR. Validação para o português do International Consultation on Incontinence Questionnaire - Short Form (ICIQ-SF). Rev Saúde Pública 2004; 38:438-44.

25. Queijo AF. Tradução para o português e validação de um instrumento de medida de carga de trabalho de enfermagem em Unidade de Terapia Intensiva: Nursing Activities Score (N.A.S.) [Dissertação de Mestrado]. Ribeirão Preto: Escola de Enfermagem de Ribeirão Preto, Universidade de São Paulo; 2002.

26. Nunes B. Tradução para o português e validação de um instrumento de medida de gravidade na UTI: TISS - 28 Therapeutic Intervention Scoring Sistem [Dissertação de Mestrado]. Ribeirão Preto: Escola de Enfermagem de Ribeirão Preto, Universidade de São Paulo; 2000.

27. De Micheli D. Uso de drogas por adolescentes: adaptação e validação de um instrumento de triagem (DUSI) e estudo das razões do uso inicial [Tese de Doutorado]. São Paulo: Escola Paulista de Medicina, Universidade Federal de São Paulo; 2000.

28. Lino MM. Satisfação profissional entre enfermeiras de UTI: adaptação transcultural do Index of Work Satisfaction (IWS) [Dissertação de Mestrado]. Ribeirão Preto: Escola de Enfermagem de Ribeirão Preto, Universidade de São Paulo; 1999.

29. Kimura M. Tradução para o português e validação do Quality of Life Index, de Ferrans e Powers [Tese de Livre Docência]. Ribeirão Preto: Escola de Enfermagem de Ribeirão Preto, Universidade de São Paulo; 1999.

30. Gutierrez BAO. Tradução para o português e descrição do processo de validação do Seatle Obstructive Lung Disease Questionnaire [Dissertação de Mestrado]. Ribeirão Preto: Escola de Enfermagem de Ribeirão Preto, Universidade de São Paulo; 2000 .
31. Bighetti F. Tradução e validação do Eating Attitudes Test (EAT-26) em adolescentes do sexo feminino na cidade de Ribeirão Preto-SP [Dissertação de Mestrado]. Ribeirão Preto: Escola de Enfermagem de Ribeirão Preto, Universidade de São Paulo; 2003.

32. Lino VTS, Pereira SRM, Camacho LAB, Ribeiro Filho ST, Buksman S. Adaptação transcultural da Escala de Independência em Atividades da Vida Diária (Escala de Katz). Cad Saúde Pública 2008; 24:103-12.

33. Souza AC, Magalhães LC, Teixeira-Salmela LF. Adaptação transcultural e análise das propriedades psicométricas da versão brasileira do Perfil de Atividade Humana. Cad Saúde Pública 2006; 22:2623-36.

34. Araujo RB, Oliveira MS, Mansur MA. A validação brasileira do Questionnaire of Smoking Urges. Cad Saúde Pública 2006; 22:2157-67.

35. Remor E, Milner-Moskovics J, Preussler G. Adaptação brasileira do "Cuestionario para la Evaluación de la Adhesión al Tratamiento Antiretroviral". Rev Saúde Pública 2007; 41:685-94.

36. Pedroso RS, Oliveira MS, Moraes JFD. Tradução, adaptação e validação da versão brasileira da escala Marijuana Expectancy Questionnaire. Cad Saúde Pública 2007; 23:63-73.

37. Pesce RP, Assis SG, Avanci JQ, Santos NC, Malaquias JV, Carvalhaes R. Adaptação transcultural, confiabilidade e validade da escala de resiliência. Cad Saúde Pública 2005; 21:436-48.

38. Reichenheim ME, Moraes CL. Operacionalização de adaptação transcultural de instrumentos de aferição usados em epidemiologia. Rev Saúde Pública 2007; 41:665-73.

39. Herdman M, Fox-Rushby J, Badia X. 'Equivalence' and the translation and adaptation of health-related quality of life questionnaires. Qual Life Res 1997; 6:237-47.

40. Perneger TV, Leplège A, Etter JF. Cross-cultural adaptation of a psychometric instrument: two methods compared. J Clin Epidemiol 1999; 52:1037-46.

41. Moraes CL, Hasselmann MH, Reichenheim ME. Adaptação transcultural para o português do instrumento Revised Conflict Tactics Scales (CTS2) utilizado para identificar violência entre casais. Cad Saúde Pública 2002; 18163-76.

42. Grassi-Oliveira R, Stein LM, Pezzi JC. Tradução e validação de conteúdo da versão em português do Childhood Trauma Questionnaire. Rev Saúde Pública 2006; 40:249:55.

43. Paixão Jr. CM, Reichenheim ME, Moraes CL, Coutinho ESF, Veras RP. Adaptação transcultural para o Brasil do instrumento Caregiver Abuse Screen (CASE) para detecção de violência de cuidadores contra idosos. Cad Saúde Pública 2007; 23:2013-22.

44. Pereira LSM, Marra TA, Faria CDCM, Pereira DS, Martins MAA, Dias JMD, et al. Adaptação transcultural e análise da confiabilidade do Southampton Assessment of Mobility para avaliar a mobilidade de idosos brasileiros com demência. Cad Saúde Pública 2006; 22:2085-95.

Recebido em 22/Nov/2007

Versão final reapresentada em 27/Fev/2008 Aprovado em 28/Abr/2008 